\title{
Archives
}

\section{Accart, Jean-Philippe. Regards croisés sur les métiers des sciences de l'information : Bibliothèques, Archives, Documentation, Musées. Mont-Saint-Aignan : éditions KLOG, 2014, 119 pages}

\section{Sabine Mas}

Volume 46, numéro 1, 2016

URI : https://id.erudit.org/iderudit/1035726ar

DOI : https://doi.org/10.7202/1035726ar

Aller au sommaire du numéro

Éditeur(s)

Association des archivistes du Québec (AAQ)

ISSN

0044-9423 (imprimé)

2369-9256 (numérique)

Découvrir la revue

Citer ce compte rendu

Mas, S. (2016). Compte rendu de [Accart, Jean-Philippe. Regards croisés sur les métiers des sciences de l'information: Bibliothèques, Archives, Documentation, Musées. Mont-Saint-Aignan : éditions KLOG, 2014, 119 pages]. Archives, 46(1), 107-109. https://doi.org/10.7202/1035726ar d'utilisation que vous pouvez consulter en ligne.

https://apropos.erudit.org/fr/usagers/politique-dutilisation/ 


\section{COMPTE RENDU}

Accart, Jean-Philippe. Regards croisés sur les métiers des sciences de l'information: Bibliothèques, Archives, Documentation, Musées. Mont-Saint-Aignan : éditions KLOG, 2014, 119 pages.

\section{Sabine Mas}

Professeure agrégée, Université de Montréal

L'auteur de cet essai, Jean-Philippe Accart, est responsable de la bibliothèque de l'École Hôtelière de Lausanne en Suisse. Il s'intéresse depuis de nombreuses années à différents métiers, dont celui de documentaliste et de bibliothécaire. Cette onzième monographie est le fruit d'années d'expérience comme formateur et professionnel et veut rendre compte d'une réflexion comparative sur les métiers de l'information (archivistique, bibliothéconomie, documentation, muséologie) dans ce début du $21^{\mathrm{e}}$ siècle qui constitue, selon l'auteur, un temps de changement et d'évolution importante pour ceux-ci. L'ouvrage est préfacé par Carol Couture qui souligne la pertinence du sujet dans un contexte de mise en commun réalisée ou qu'envisagent de réaliser plusieurs institutions culturelles ou d'enseignement.

En introduction, l'auteur rappelle la présence et l'intégration des institutions de culture et de mémoire (archives, bibliothèques, documentation et musées) au cœur de la vie citoyenne et à chaque échelon administratif (municipal, national, etc.). Ces lieux favorisent le plus souvent gratuitement l'accès à l'information, à la culture, à la technologie et offrent des espaces de travail et de discussion en dehors de la sphère familiale ou professionnelle. Les sphères professionnelle, privée et numérique possèdent elles aussi leurs lieux de culture et de mémoire et sont investies par la technologie numérique qui constitue un point de convergence fort des métiers de l'information.

Dans le premier chapitre, l'auteur aborde le sujet des missions communes des bibliothèques, archives, documentation et musées soit la préservation, la conservation et la diffusion du patrimoine et des archives. Par conséquent, les archives partagent avec les bibliothèques et les musées une vocation culturelle, scientifique et administrative. Il s'agit de lieux d'apprentissage, de connaissances et de mémoire ouverts à des publics différents, et favorisant le rapprochement intergénérationnel.

Le chapitre suivant accorde une place à l'utilisateur des services et collections, qui se situe au cœur des métiers et en justifie l'existence. Les professionnels de l'information doivent répondre aux besoins d'information d'utilisateurs généralement peu critiques envers les sources. Les utilisateurs se tournent vers les institutions documentaires pour être accompagnés quand la recherche est trop complexe et que les résultats trouvés sur Internet sont insuffisants ou non pertinents. Enfin, ces utilisateurs s'attendent désormais à une offre d'utilisation à distance des services sur le Web.

Le troisième chapitre présente les trois caractéristiques communes des métiers étudiés (archives, bibliothèques, documentation, musées): 1) ils traitent des objets 
(documents, objets, tableaux, etc.) qui suivent une chaîne de traitement similaire à travers les fonctions d'acquisition, de traitement et de diffusion, 2) ils répondent à une demande du public, et 3) ils ont une composante technologique importante. L'auteur s'attarde ensuite sur les aspects historiques des trois métiers principaux (bibliothécaires, archivistes, documentalistes), présente les lieux et niveaux de formations en France puis commente une cartographie de la multiplicité des métiers de l'information regroupés en sept grandes familles par l'Association des professionnels de l'information et de la documentation (ADBS) et qui oblige à repenser la vision traditionnelle de ces métiers.

Le quatrième chapitre fait état des pratiques archivistiques, bibliothéconomiques, documentaires et muséales. Dans un premier temps, l'auteur fait un survol des différences et des pratiques similaires ou complémentaires entre les domaines. Les deux notions de polyvalence et d'hybridation sont représentatives et emblématiques des métiers de l'information décrits ici, et constituent certainement une voie possible pour leur avenir. L'auteur souligne que la formation initiale a bien compris cette complémentarité et croise souvent les matières d'études.

Le cinquième chapitre de cet ouvrage est consacré à certains aspects de la technologie liés aux métiers des sciences de l'information et qui présentent des implications sociales (logiciels, progiciels, portails, SIG, infonuagique, numérisation, open access, réseaux sociaux, humanités numériques). Cette technologie numérique, même si elle remet en question certains principes et certaines pratiques, pourrait être l'occasion pour les métiers de l'information de se réinventer. Selon l'auteur, le monde de l'information devenu trop complexe, demande l'alliance de plusieurs métiers pour savoir l'utiliser et l'exploiter au mieux.

Le dernier chapitre aborde les questions de coopération, de collaboration et de partenariat entre les métiers de l'information qui conservent un patrimoine appartenant à une même communauté avec des moyens ou des actions très proches. L'auteur présente quelques pratiques exemplaires et des ressources électroniques produites en collaboration. Pour terminer, l'auteur mentionne les risques et les bénéfices attendus de la coopération entre les institutions culturelles.

Le sujet de l'ouvrage n'est pas nouveau; la tendance de rapprochement ou de convergence entre les différents métiers de l'information a bien été documentée depuis plusieurs décennies. Comme le rappelle très justement Carol Couture en préface, dès la fin des années 1980, le Programme général d'information (PGI) de l'UNESCO faisait la promotion d'une "harmonisation" entre les programmes de formation des spécialistes des sciences de l'information, soit les bibliothécaires, les documentalistes et les archivistes à travers des colloques et des publications. Plusieurs universités canadiennes intègrent l'archivistique et la bibliothéconomie dans les cursus en sciences de l'information dès le début des années 1980 et 1990. Plus récemment, les iSchools intègrent toutes les formations qui traitent de la gestion de l'information. Certains pays comme les États-Unis ou le Canada ne voient même pas de différences fondamentales entre documentaliste-bibliothécaire ou documentaliste-archiviste. Quant à elle, la convergence entre les institutions patrimoniales ou les LAM (Libraries Archives Museum) est une forte tendance depuis les années 2000 avec notamment les rapports de l'OCLC et de l'IFLA en 2008 à ce sujet. 
Il s'agit d'une synthèse sur la question des métiers de l'information que beaucoup de professionnels pratiquent à un moment ou un autre durant leur carrière. L'auteur fait un survol des missions des institutions, de leur clientèle, des professionnels, des pratiques, de la technologie à travers une bonne connaissance des domaines de la documentation et des bibliothèques et "quelques connaissances" sur l'archivistique et la muséologie dans un contexte principalement français ou suisse. Par conséquent, les archivistes ne liront pas ce livre pour en apprendre davantage sur les obstacles à relever, les enjeux ou les effets de la convergence intra-institution ou extra-institution qui y sont peu développés; ils liront ce livre pour découvrir ou redécouvrir les disciplines "sœurs" que sont la bibliothéconomie, la documentation et la muséologie et les possibilités ou exemples de projets de collaboration, coopération et de partenariat. Par conséquent, certains chapitres seront lus plus rapidement que d'autres selon les connaissances et expériences personnelles du lecteur.

Toutefois, le sujet de l'ouvrage est devenu incontournable en raison des technologies du numérique et des missions de plus en plus nombreuses des institutions patrimoniales dans un contexte de restrictions budgétaires. L'utilisation croissante d'Internet à la fin des années 1990 pour la recherche en ligne oblige à changer et à moderniser les méthodes de travail. La lecture de cet ouvrage nous convainc de l'apport des technologies du numérique qui offrent l'opportunité de rapprocher voire de rassembler à un même endroit (Internet) ce qui a été longtemps physiquement séparé afin de répondre au rêve de tout utilisateur : l'utopie de "l'archive totale" soit de retrouver à un même endroit l'ensemble des documents (archives, livres, etc.), artefacts, objets, etc. relatif à un même sujet. Par ailleurs, l'auteur de l'ouvrage rappelle qu'il peut être rentable non seulement au plan économique, mais aussi aux plans scientifique et professionnel de mettre en commun les ressources, les connaissances et les pratiques comme l'ont fait Bibliothèque et Archives Canada en 2004 et Bibliothèque et Archives nationales du Québec en 2006 avec deux visions différentes cependant, la première institution prônant "l'intégration", et la deuxième "l'harmonisation", plus respectueuse des spécificités des professions et des disciplines.

Pour terminer, l'originalité de l'ouvrage repose sans aucun doute sur les nombreux témoignages de professionnels qui ont été sollicités et qui figurent tout au long de cet ouvrage. À ce sujet, il est à souligner le témoignage perspicace d'une bibliothécaire travaillant pour le service d'archives de la Ville de Lausanne, Fabienne Chuat, sur les convergences et différences des domaines archives et bibliothèques. 